\title{
Role of Quality Management System in Enhancing the Performance in Manufacturing Industries
}

\author{
G Sivaramakrishnan* and S Giridhara ${ }^{\dagger}$
}

\begin{abstract}
After globalization in the 1990s, the standardization process in manufacturing industries has gained momentum. In this study, an attempt has been made to identify the role of Quality Management System (QMS) in enhancing performance in an Indian manufacturing industry, with M/S Ramdevs Motors, a pumps manufacturing industry, as the place of this study. Performance measures like, Increase in financial performance, productivity, etc. have been considered in this study. An opinion survey type questionnaire was used to collect data from the organization. The performance of the company before and after the implementation of QMS was collected, processed and analysed using statistical tools. The results of the study reveal that the role of QMS has significance in enhancing the performance of the organization but has no significance in financial performance of the organization.
\end{abstract}

Keywords: QMS - Quality Management Systems - Enhancing Performance of Manufacturing Industries

\footnotetext{
*Vels University Chennai, ganshiv_g@yahoo.co.in

† Quality Council of India, New Delhi and Aakarshna Quality Management Services, Coimbatore, India; 607014@gmail.com
} 


\section{Introduction}

The management of the organization in this study opts to implement a Quality Management System as per ISO 9001 standard with the following considerations:

- Customers demand ISO 9001 certificate.

- To supply consistent quality products to the market and to sustain the product quality.

- To have a control on the manufacturing processes.

- To have a control on the internal documents (Example: design drawings).

- To do manufacturing process in a systematic way.

The organization appointed a consultant to implement QMS and the organization is certified to ISO 9001:2008 QMS certification after effective QMS Audit. The management and entire team are fully involved in the ISO 9001 implementation and the organization maintains the Quality Management System as per ISO 9001. However, the management is keen to know the following:

- Customer demands Quality Management System certification, but the organization faces recurring expenses to maintain QMS certification. The management needs a justification for this recurring expenditure on the choice of QMS Certification option.

- The management is also keen to understand, whether QMS is really beneficial to their organization in improving performance towards achieving their organizational objectives.

In broad perspective, from a survey data (Source : www.iso.org) from International Organization for Standardization, the total number of ISO 9001 certified companies in the world as on December 2012 is 11,01,272. Number of ISO 9001 certified in India is 29,402 which is $2.7 \%$ of the world certification which reflects the significance of QMS in improving the performance of manufacturing industries as these manufacturing industries are the key contributors for the Economic growth of India and the Manufacturing Industries contribute 15\% (as on 2012) to the GDP of India. 
Sivaramakrishnan and S Giridhara Role of Quality Management System

\section{Limitations}

This study is limited to Manufacturing Industries only and the researchers have only considered $M / S$ Ramdevs Motors as the place of study.

This study is limited to the role of Quality Management system as per ISO 9001 standard. (Note: Quality Management is a part of overall management) This study does not cover other management certification standards like Environmental Management System as per ISO 14001, Occupational Health and Safety standard as per BS OHSAS 18001 etc.

Since certain requirements are not covered in the ISO 9001:2008 standard, while implementing QMS and during the QMS certification process in the manufacturing sector, those requirements are not covered, for example:

- Finance requirements

- Accounting practices and processes

- Payment transactions

- Payrolls/ESI/PF requirements

- Formation of company, board of directors, memorandum of associations

- Environmental requirements/conditions and norms

- Safety requirements/conditions

- Share holders requirements

- Employee's expectations

- Social responsibility

- Suppliers expectations (example: complying to supplier payments)

\section{Objective of the Study}

The objective of this study is to find the role of Quality Management System in improving the following organizational objectives:

- Financial performance.

- Quality \& productivity.

- Customer Relation Management.

- Employee performance. 
- Document and record control.

- Overall performance and

- To provide recommendations/suggestions to enhance the Quality Management System.

\section{Literature Review}

Esin Sadikoglu and Hilal Olcay (2014) have observed that TQM index can be positively related to performance measures - Market, finance, Inventory management, Innovation, and improve performance of the enterprise as a whole.

Stella E. Garcia (1999) has indicated that eight of the ten dependent variables demonstrate a positive relationship between organizational performance and quality management ${ }^{7}$.

Dale H.Besterfield, Carol Besterfield, et al. analysed the Benefits of ISO registration and found that significant improvement could be brought in

- Internal quality - measured by the generation of rework, scrap, and non-conformance.

- External quality - measured by customer acceptance value without inspection, sale return and claims on defective product.

- Time performance is measured by delivery of products on time to market.

- Scrap, Rework, External Non-conformance are the indices of Cost of poor quality.

The North Town of automobile dealers of Toronto, Ontario have observed that ISO registration has positive implication that the customer loyalty and satisfaction has improved by $20 \%$ and good will by customer recommending the dealership by 55\%. The experience of United Airlines for ISO registration is that it has reduced the overhaul cycle time of engine from 120 days to 60 days.

\section{Research Gap}

While similar types of studies have been conducted in many places of the world, researchers have not focused much on the industry in India. Especially, the Small scale Manufacturing sector in India is 
yet to be explored. Hence, there is a research gap and a need for this study with M/S Ramdevs Motors as the place of study.

\section{Methodology}

The time taken for this study is one year. Opinions of employee and Management Team have been collected in a questionnaire (these questionnaires have been given as tables from the section 7.1 to 7.9 below). Data related to performance of the organization before implementing QMS and one year after implementing QMS have been collected in the questionnaire.

The employees, staff and Chief Executive officer (CEO) are the target the respondents, a questionnaire is submitted and their opinion is collected back.

The In-charge/Head of the department of the following functions were interviewed.

- Design

- Production \& Machine Maintenance

- Quality

- Purchase

- Finance

Two senior operators from production were interviewed. CEO of the organization was interviewed. Number of respondents used in the study was 8 (eight). Opinions given by these respondents are summarised in the questionnaire (these questionnaires have been given as tables from the section 7.1 to 7.9 below).

Respondents for the survey are chosen based on their presence before and after implementing QMS. Staff and employee involved in the development of QMS and able to recognize the changes before and after implementing QMS are chosen for this study.

Research design method followed in this study is "Exploratory Study"

The Sample design followed in this study is "Non-Probability design" and Census sampling technique is followed as sampling method. The management and employees of the company is considered as the sampling unit. 
Type of collected data through the Questionnaire is a qualitative or attributes data. The following are the attribute data in this study.

\author{
E-Efficient \\ G-Good \\ M-Moderate \\ LM-Less than Moderate \\ P-Poor
}

Data collected from the questionnaire is processed and further analysed by the following two methods.

(i) \% Analysis: In this method, \% data is evaluated before and after implementation of QMS. Difference between \% before implementation and after implementation QMS shows the improvement.

(ii) Chi-Square $\left(X^{2}\right)$ test: This test is carried out using computerised Statistical package.

\title{
Tools Used for Analysis
}

Since type of data used is qualitative/attribute data, Chi-Square $\left(X^{2}\right)$ test is used as statistical tool to analyse the data.

Chi-Square $\left(\chi^{2}\right)$ test: A chi square test, called "test of association," is a statistical tool that measures the association between discrete variables or data. It is computed based on a the comparison of the number of observed counts with the number of expected counts to determine whether there is a difference in output counts based on the input category. This test is used for Qualitative/Attribute data in which are numbers (counts), for example number of errors in a manufactured product or opinions like good or bad. Critical Chisquared value is $p=0.05$. If $\mathbf{P}$ value is less than or equal to 0.05 then test of association between two group of data is significant.

Before and after implementation of QMS is evaluated through $\%$ improvement analysis also. Difference between \% before implementation and after implementation QMS shows the improvement.

However significance level of improvement is finally taken from the Chi-Square $\left(\chi^{2}\right)$ test result. 
Sivaramakrishnan and S Giridhara Role of Quality Management System

\section{Data Analysis and Interpretation}

\section{QMS Vs Financial Performance}

Table 1QMS Vs Financial performance data: \% Improvement Analysis-

\begin{tabular}{|c|c|c|c|c|c|c|c|c|c|c|c|}
\hline \multirow{2}{*}{$\frac{y}{a}$} & \multirow[t]{2}{*}{$\begin{array}{l}\text { Performance } \\
\text { aspects }\end{array}$} & \multicolumn{5}{|c|}{$\begin{array}{l}\text { Status Before QMS } \\
\text { Implementation }\end{array}$} & \multicolumn{5}{|c|}{$\begin{array}{c}\text { Status : Within } 3 \text { years } \\
\text { After QMS } \\
\text { implementation }\end{array}$} \\
\hline & & E & G & $\mathrm{M}$ & LM & $\mathrm{P}$ & $\mathrm{E}$ & $\mathrm{G}$ & $\mathrm{M}$ & LM & $\mathrm{P}$ \\
\hline \multicolumn{2}{|c|}{$\begin{array}{l}\text { Maximum Rating } \\
\text { (B) }\end{array}$} & 100 & 80 & 60 & 20 & 0 & 100 & 80 & 60 & 20 & 0 \\
\hline 1. & $\begin{array}{l}\text { Investment } \\
\text { for QMS Vs } \\
\text { Organization } \\
\text { growth }\end{array}$ & & & & & $\bullet$ & & $\bullet$ & & & \\
\hline 2. & $\begin{array}{l}\text { Return on } \\
\text { investment- } \\
\text { General }\end{array}$ & & & & & • & & & $\bullet$ & & \\
\hline 3. & $\begin{array}{l}\text { Return on } \\
\text { investment- } \\
\text { QMS }\end{array}$ & & & & & $\bullet$ & & $\bullet$ & & & \\
\hline 4. & $\begin{array}{l}\text { Market share- } \\
\text { Domestic }\end{array}$ & & & & $\bullet$ & & & & $\bullet$ & & \\
\hline 5. & $\begin{array}{l}\text { Market share- } \\
\text { International }\end{array}$ & & & & & • & & & & & $\bullet$ \\
\hline 6. & $\begin{array}{l}\text { Increase in } \\
\text { turnover }\end{array}$ & & & $\bullet$ & & & & & $\bullet$ & & \\
\hline 7. & $\begin{array}{l}\text { Increase in } \\
\text { profits }\end{array}$ & & & & & • & & & & $\bullet$ & \\
\hline 8 & Total Items A & 0 & 0 & 1 & 1 & 5 & 0 & 2 & 3 & 1 & 1 \\
\hline $\begin{array}{l}9 \\
.\end{array}$ & $\begin{array}{l}\text { Maximum } \\
\text { score }(C)\end{array}$ & & & $100=$ & & & & & $100=$ & & \\
\hline 1 & Obtained & & & & & & & & & & \\
\hline 0. & $\begin{array}{l}\text { score } \\
\mathrm{D}=\Sigma(\mathrm{AXB})\end{array}$ & & & 80 & & & & & 360 & & \\
\hline 1 & $\%$ Obtained & & & & & & & & & & \\
\hline 1. & $\begin{array}{l}\text { score } \\
(\mathrm{DX100)/C}\end{array}$ & & & $1.4 \%$ & & & & & $51.4 \%$ & & \\
\hline
\end{tabular}

\section{Findings/Inferences: QMS Vs Financial Performance}

Percentage Analysis shows, 40\% improvement in the financial performance is found after QMS implementation. However chisquare test shows $P$ value $=0.129$ which is not a significant change. Hence, it is interpreted that, the role of QMS has no significance in improving the financial performance of the organization. 


\section{QMS Vs Productivity}

Table 2 QMS Vs Productivity data: \% Improvement Analysis -

\begin{tabular}{llllllllll}
\hline $\begin{array}{l}\text { S1. } \\
\text { No }\end{array} \quad \begin{array}{l}\text { Performance } \\
\text { aspects }\end{array}$ & \multicolumn{3}{c}{$\begin{array}{c}\text { Status Before QMS } \\
\text { Implementation }\end{array}$} & \multicolumn{3}{c}{ Status : Within 3 years } \\
After QMS implementation
\end{tabular}

\section{Findings/Inferences: QMS Vs Productivity}

Percentage Analysis shows, $66.7 \%$ improvement in the productivity is found after QMS implementation. Chi-square test shows $P$ value $=0.007$, which is a significant change. Hence, it is interpreted that, the role of QMS has significance in improving the productivity of the organization. 
Sivaramakrishnan and S Giridhara Role of Quality Management System

\section{QMS Vs Quality Improvement}

Table 3 QMS Vs Quality Improvement: \% Improvement Analysis-

\begin{tabular}{|c|c|c|c|c|c|c|c|c|c|c|c|}
\hline \multirow[t]{2}{*}{$\begin{array}{l}\text { Sl. } \\
\text { No }\end{array}$} & \multirow[t]{2}{*}{ Performance aspects } & \multicolumn{5}{|c|}{$\begin{array}{c}\text { Before QMS } \\
\text { Implementation }\end{array}$} & \multicolumn{5}{|c|}{$\begin{array}{c}\text { Within } 3 \text { years } \\
\text { After QMS } \\
\text { implementation }\end{array}$} \\
\hline & & E & G & M & LM & $\mathrm{P}$ & E & $\mathrm{G}$ & M & LM & $\mathrm{P}$ \\
\hline & Maximum Rating (B) & 100 & 80 & 60 & 20 & 0 & 100 & 80 & 60 & 20 & 0 \\
\hline 1. & $\begin{array}{l}\text { Product Design } \\
\text { improvements }\end{array}$ & & & & $\bullet$ & & & • & & & \\
\hline 2. & $\begin{array}{l}\text { Control of } \\
\text { drawings/quality } \\
\text { plans }\end{array}$ & & & & & & $\bullet$ & & & & \\
\hline 3. & $\begin{array}{l}\text { Improvement in } \\
\text { incoming materials } \\
\text { inspection/product } \\
\text { testing process }\end{array}$ & & & & $\bullet$ & & & & $\bullet$ & & \\
\hline 4. & $\begin{array}{l}\text { Internal Non } \\
\text { conformance }\end{array}$ & & & & & $\bullet$ & & $\bullet$ & & & \\
\hline 5. & Process control & & & & $\bullet$ & & & $\bullet$ & & & \\
\hline 6. & Customer complaints & & & & & $\bullet$ & & $\bullet$ & & & \\
\hline 7. & $\begin{array}{l}\text { field failures and } \\
\text { returns }\end{array}$ & & & & $\bullet$ & & & & $\bullet$ & & \\
\hline 8. & Cost of poor quality & & & & & $\bullet$ & & & $\bullet$ & & \\
\hline 9. & $\begin{array}{l}\text { Corrective action for } \\
\text { internal NCs }\end{array}$ & & & & & $\bullet$ & & & • & & \\
\hline 10. & $\begin{array}{l}\text { 5s-house keeping/ } \\
\text { cleanliness }\end{array}$ & & & & & & $\bullet$ & & & & \\
\hline 11. & $\begin{array}{l}\text { Identification, } \\
\text { method of storage } \\
\text { and Traceability of } \\
\text { parts }\end{array}$ & & & & $\bullet$ & & & $\bullet$ & & & \\
\hline 12. & $\begin{array}{l}\text { Supplier quality } \\
\text { (incoming NC parts) }\end{array}$ & & & & & $\bullet$ & & & $\bullet$ & & \\
\hline 13. & $\begin{array}{l}\text { Supplier's delivery } \\
\text { performance }\end{array}$ & & & & • & & & & & • & \\
\hline 14. & $\begin{array}{l}\text { Supplier PO tracking } \\
\text { system }\end{array}$ & & & & & $\bullet$ & & & $\bullet$ & & \\
\hline 15. & $\begin{array}{l}\text { Defect prevention/ } \\
\text { implementation of } \\
\text { preventive/proactive } \\
\text { active controls }\end{array}$ & & & & & $\bullet$ & & & $\bullet$ & & \\
\hline Tota & Items A & 0 & 0 & 0 & 6 & 9 & 2 & 5 & 7 & 1 & \\
\hline $\operatorname{Max}$ & num score (C) & & $15 X$ & $00=1$ & & & & $15 X$ & $00=-$ & & \\
\hline Obta & ned score $\mathrm{D}=\Sigma(\mathrm{AXB})$ & & & 20 & & & & & 040 & & \\
\hline $\begin{array}{l}\% \mathrm{O} \\
(\mathrm{DX}\end{array}$ & $\begin{array}{l}\text { tained score } \\
\text { O) /C }\end{array}$ & & & $0 \%$ & & & & & $9.3 \%$ & & \\
\hline
\end{tabular}




\section{Findings/Inferences: QMS Vs Quality Improvement}

Percentage Analysis shows, $61.3 \%$ improvement in the quality is found after QMS implementation. Chi-square test shows $\mathrm{P}$ value $=0.000$, which is a significant change. Hence, it is interpreted that, the role of QMS has significance in quality improvement of the organization.

\section{QMS Vs Employee Performance}

Table 4QMS Vs Employee Performance: \% Improvement Analysis-

\begin{tabular}{|c|c|c|c|c|c|c|c|c|c|c|c|}
\hline \multirow[t]{2}{*}{$\begin{array}{l}\text { Sl. } \\
\text { No. }\end{array}$} & \multirow[t]{2}{*}{$\begin{array}{l}\text { Performance } \\
\text { aspects }\end{array}$} & \multicolumn{5}{|c|}{$\begin{array}{c}\text { Before QMS } \\
\text { Implementation }\end{array}$} & \multicolumn{5}{|c|}{$\begin{array}{c}\text { Within } 3 \text { years } \\
\text { After QMS } \\
\text { implementation }\end{array}$} \\
\hline & & $E$ & G & $\mathrm{M}$ & LM & $\mathrm{P}$ & $E$ & G & $\mathrm{M}$ & LM & $\mathrm{P}$ \\
\hline & Maximum & 100 & 80 & 60 & 20 & 0 & 100 & 80 & 60 & 20 & 0 \\
\hline 1. & $\begin{array}{l}\text { Individual } \\
\text { performance } \\
\text { of employees }\end{array}$ & & & & $\bullet$ & & & & $\bullet$ & & \\
\hline 2. & $\begin{array}{l}\text { Department } \\
\text { wise performance }\end{array}$ & & & & $\bullet$ & & & & $\bullet$ & & \\
\hline 3. & $\begin{array}{l}\text { Employee } \\
\text { involvement }\end{array}$ & & & & $\bullet$ & & & & $\bullet$ & & \\
\hline 4. & $\begin{array}{l}\text { Employee } \\
\text { satisfaction }\end{array}$ & & & & $\bullet$ & & & & $\bullet$ & & \\
\hline 5. & $\begin{array}{l}\text { Employee } \\
\text { awareness on } \\
\text { QMS }\end{array}$ & & & & & $\bullet$ & & $\bullet$ & & & \\
\hline 6. & $\begin{array}{l}\text { Effectiveness } \\
\text { of employee } \\
\text { training on } \\
\text { QMS }\end{array}$ & & & & & $\bullet$ & & & $\bullet$ & & \\
\hline \multicolumn{2}{|c|}{ Total Items A } & 0 & 0 & 0 & 4 & 2 & 0 & 1 & 5 & 0 & 0 \\
\hline \multicolumn{2}{|c|}{ Maximum score $(\mathrm{C})$} & & \multicolumn{3}{|c|}{$6 \times 100=600$} & & & \multicolumn{3}{|c|}{$6 \times 100=600$} & \\
\hline \multicolumn{2}{|c|}{$\begin{array}{l}\text { Obtained score } \\
\mathrm{D}=\Sigma(\mathrm{A} X \mathrm{~B})\end{array}$} & & \multicolumn{3}{|c|}{80} & & & \multicolumn{3}{|c|}{380} & \\
\hline \multicolumn{2}{|c|}{$\begin{array}{l}\text { \% Obtained score } \\
(\mathrm{DX} 100) / \mathrm{C}\end{array}$} & & \multicolumn{3}{|c|}{$13.3 \%$} & & & \multicolumn{3}{|c|}{$63.3 \%$} & \\
\hline
\end{tabular}

\section{Findings/Inferences: QMS Vs Employee Performance}

Percentage Analysis shows, 50\% improvement in the employee performance is found after QMS implementation. Chi-square test shows $\mathrm{P}$ value $=0.05$, which is a significant change. Hence, it is interpreted that, the role of QMS has significance in improving the employee performance of the organization. 
Sivaramakrishnan and S Giridhara Role of Quality Management System

\section{QMS Vs Customer Relation Management}

Table 5 QMS Vs Customer relation management: \% Improvement Analysis -

\begin{tabular}{|c|c|c|c|c|c|c|c|c|c|c|c|}
\hline \multirow[t]{2}{*}{$\begin{array}{l}\text { Sl. } \\
\text { No. }\end{array}$} & \multirow[t]{2}{*}{$\begin{array}{l}\text { Performance } \\
\text { aspects }\end{array}$} & \multicolumn{5}{|c|}{$\begin{array}{c}\text { Before QMS } \\
\text { Implementation }\end{array}$} & \multicolumn{5}{|c|}{$\begin{array}{l}\text { Within } 3 \text { years } \\
\text { After QMS } \\
\text { implementation }\end{array}$} \\
\hline & & $\mathrm{E}$ & G & $\mathrm{M}$ & LM & $\mathrm{P}$ & $\mathrm{E}$ & G & $\mathrm{M}$ & LM & $\mathrm{P}$ \\
\hline & Maximum & 100 & 80 & 60 & 20 & 0 & 100 & 8 & 60 & 20 & 0 \\
\hline & Rating (B) & & & & & & & 0 & & & \\
\hline 1. & Customer & & & & $\bullet$ & & & 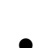 & & & \\
\hline & satisfaction level & & & & & & & & & & \\
\hline 2. & $\begin{array}{l}\text { customer relation } \\
\text { management }\end{array}$ & & & & $\bullet$ & & & $\bullet$ & & & \\
\hline 3. & $\begin{array}{l}\text { Customer order } \\
\text { tracking and } \\
\text { delivery against } \\
\text { customer order } \\
\text { tracking system }\end{array}$ & & & & & • & & $\bullet$ & & & \\
\hline \multicolumn{2}{|c|}{ Total Items A } & 0 & 0 & 0 & 2 & 1 & 0 & 3 & 0 & 0 & 0 \\
\hline \multicolumn{2}{|c|}{ Maximum score (C) } & \multicolumn{5}{|c|}{$3 \times 100=300$} & \multicolumn{5}{|c|}{$3 \times 100=300$} \\
\hline \multicolumn{2}{|c|}{ Obtained score $\mathrm{D}=\Sigma(\mathrm{AXB})$} & \multicolumn{4}{|c|}{40} & & \multicolumn{5}{|c|}{240} \\
\hline \multicolumn{2}{|c|}{$\begin{array}{l}\text { \% Obtained score } \\
\text { (DX100)/C }\end{array}$} & \multicolumn{4}{|c|}{$6.7 \%$} & & \multicolumn{5}{|c|}{$80.0 \%$} \\
\hline
\end{tabular}

\section{Findings/Inferences: QMS Vs Customer Relation Management}

Percentage Analysis shows, $73.3 \%$ improvement in the customer relation management is found after QMS implementation. Chisquare test shows $\mathrm{P}$ value $=0.05$, which is a significant change. Hence, it is interpreted that, the role of QMS has significance in improving the customer relation management of the organization. 


\section{QMS Vs Organizational Cultural Change}

Table 6QMS Vs Organizational Cultural change: \% Improvement Analysis

Graph 6: Chi-Square Test result: QMS Vs Organizational cultural change

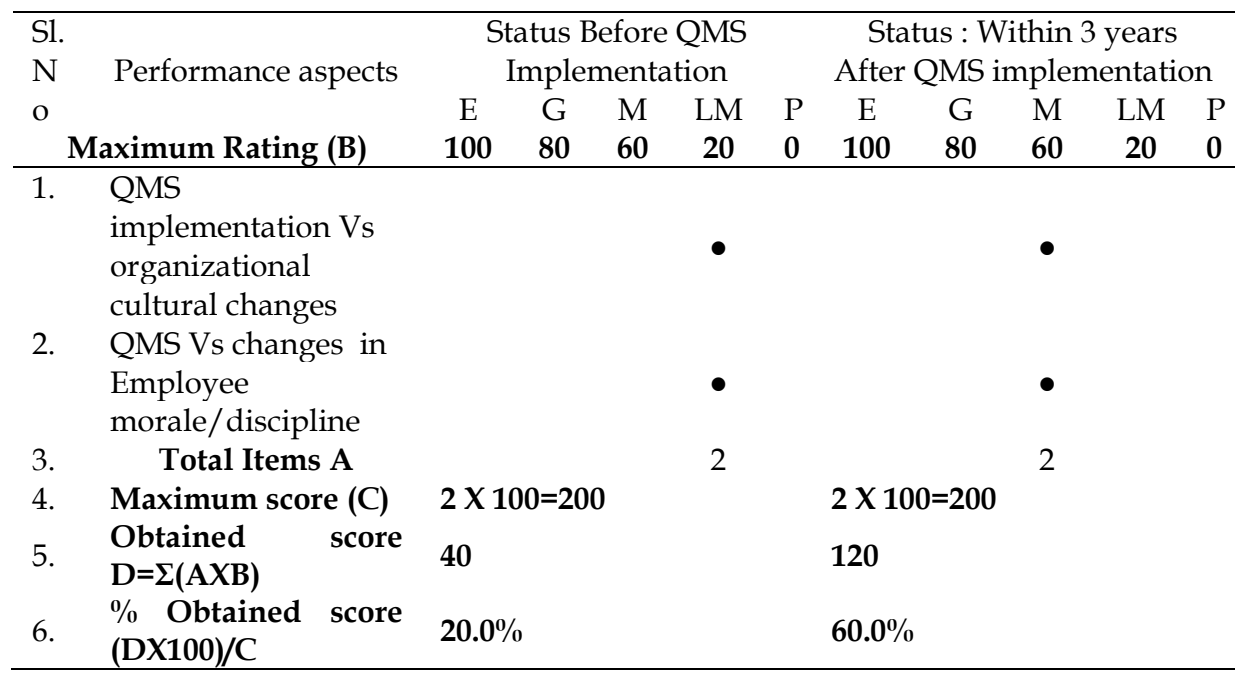

\section{Findings/Inferences: QMS Vs Organizational Cultural changes}

Percentage Analysis shows, 40\% improvement in the organizational cultural changes is found after QMS implementation. Chi-square test shows $\mathrm{P}$ value $=0.046$, which is a significant change. Hence, it is interpreted that, the role of QMS has significance in improving the organizational cultural changes. 
Sivaramakrishnan and S Giridhara Role of Quality Management System

\section{QMS Vs Document/Data/Record Controls}

Table 7QMS Vs Document/Data/Record control: \% Improvement Analysis -

Graph 7 Chi-Square Test result: QMS Vs Document/Data/Record controls

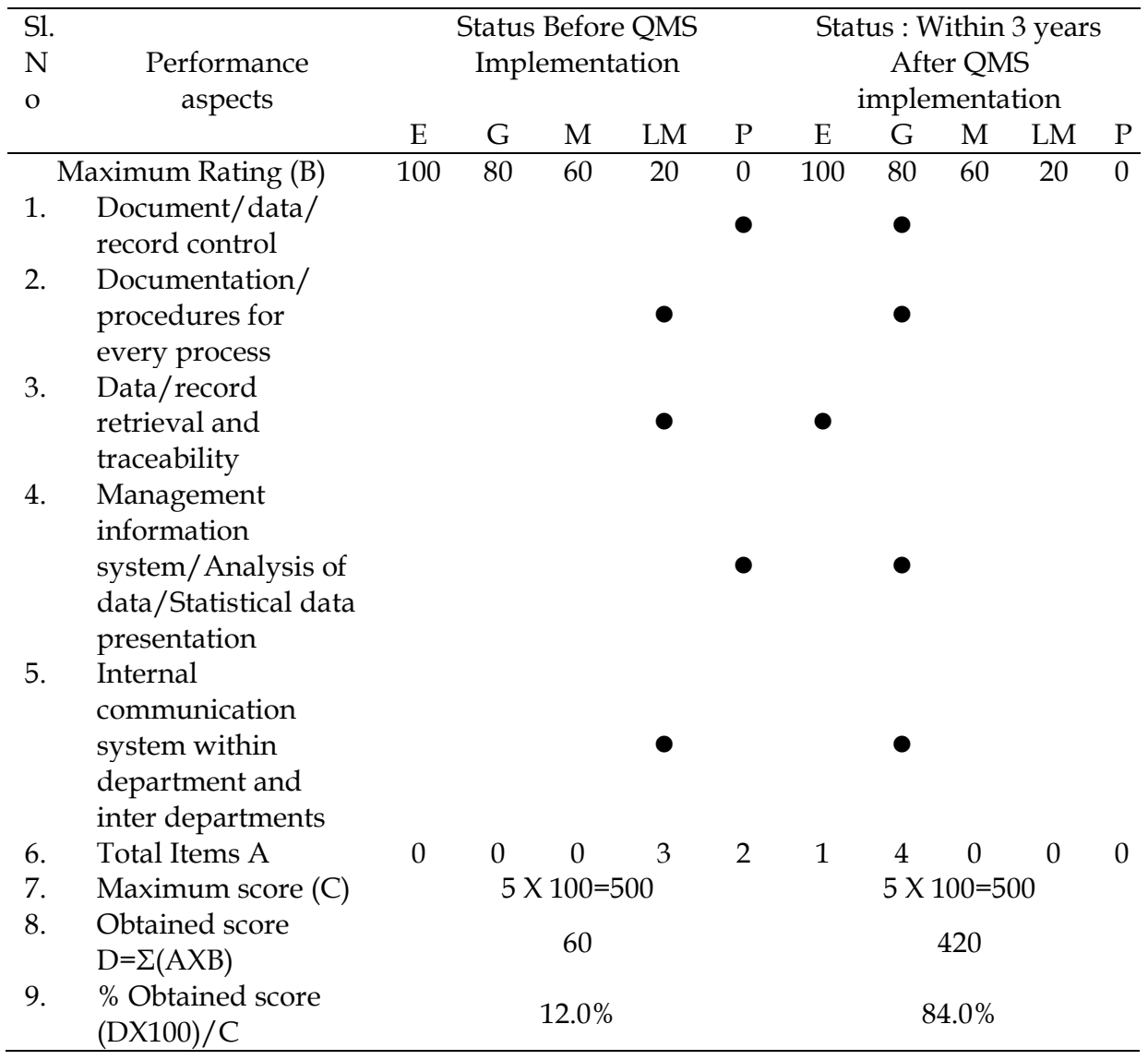

\section{Findings/Inferences: QMS Vs Document/Data/Record controls}

Percentage Analysis shows, 72\% improvement in the document/data/record controls is found after QMS implementation. Chi-square test shows $\mathrm{P}$ value $=0.019$, which is a high significant change. Hence, it is interpreted that, the role of QMS has significance in improving the performance related to document, data and record controls of the organization. 


\subsection{QMS Vs Top Management controls}

Table 8 QMS Vs Top Management controls: \% Improvement Analysis -

\begin{tabular}{|c|c|c|c|c|c|c|c|c|c|c|c|}
\hline \multirow[t]{2}{*}{$\begin{array}{l}\text { Sl. } \\
\text { No. }\end{array}$} & \multirow[t]{2}{*}{$\begin{array}{l}\text { Performance } \\
\text { aspects }\end{array}$} & \multicolumn{5}{|c|}{$\begin{array}{c}\text { Before QMS } \\
\text { Implementation }\end{array}$} & \multicolumn{5}{|c|}{$\begin{array}{l}\text { Within } 3 \text { years } \\
\text { After QMS } \\
\text { implementation }\end{array}$} \\
\hline & & $\mathrm{E}$ & G & M & LM & $\mathrm{P}$ & $\mathrm{E}$ & G & M & LM & $\mathrm{P}$ \\
\hline & Maximum & 10 & 80 & 60 & 20 & 0 & 10 & 80 & 60 & 20 & 0 \\
\hline & Rating (B) & 0 & & & & & 0 & & & & \\
\hline 1. & $\begin{array}{l}\text { Management } \\
\text { commitment } \\
\text { towards QMS }\end{array}$ & & & & $\bullet$ & & & $\bullet$ & & & \\
\hline 2. & $\begin{array}{l}\text { Management } \\
\text { policies, vision, } \\
\text { missions }\end{array}$ & & & & - & & & $\bullet$ & & & \\
\hline 3. & $\begin{array}{l}\text { Monitoring } \\
\text { department level } \\
\text { objective and } \\
\text { improvement } \\
\text { actions }\end{array}$ & & & & & $\bullet$ & & $\bullet$ & & & \\
\hline 4. & $\begin{array}{l}\text { Effectiveness } \\
\text { Internal auditing } \\
\text { process, NC }\end{array}$ & & & & & $\bullet$ & & & $\bullet$ & & \\
\hline & $\begin{array}{l}\text { Identification and } \\
\text { closing }\end{array}$ & & & & & & & & & & \\
\hline 5. & $\begin{array}{l}\text { Effectiveness of } \\
\text { Management } \\
\text { review meeting }\end{array}$ & & & & & $\bullet$ & & & $\bullet$ & & \\
\hline 6. & $\begin{array}{l}\text { Provision of } \\
\text { resources needed } \\
\text { for manufacturing } \\
\& \text { administration }\end{array}$ & & & & $\bullet$ & & & $\bullet$ & & & \\
\hline Tota & Items A & & & & 3 & 3 & & 4 & 2 & & \\
\hline $\operatorname{Max}$ & num score $(\mathrm{C})$ & & & $100=$ & & & & & $100=$ & & \\
\hline $\begin{array}{l}\text { Obta } \\
\mathrm{D}=\Sigma\end{array}$ & $\begin{array}{l}\text { hed score } \\
A X B)\end{array}$ & & & 60 & & & & & 380 & & \\
\hline $\begin{array}{l}\% \mathrm{O} \\
(\mathrm{DX}\end{array}$ & $\begin{array}{l}\text { ained score } \\
0) / C\end{array}$ & & & $10.0 \%$ & & & & & 63.3. & & \\
\hline
\end{tabular}

\section{Findings/Inferences: QMS Vs Top Management Controls}

Percentage Analysis shows, 53.3\% improvement in the top management controls is found after QMS implementation. Chisquare test shows $\mathrm{P}$ value $=0.007$, which is a significant change. Hence, it is interpreted that, the role of QMS has significance in improving the top management controls of the organization. 
Sivaramakrishnan and S Giridhara Role of Quality Management System

\subsection{QMS Vs Overall Performance}

Table 9 QMS Vs overall performance of the organization: \% Improvement Analysis -

Graph 9 Chi-Square Test result: QMS Vs Overall Organizational performance

\begin{tabular}{|c|c|c|c|c|c|c|c|c|c|c|c|}
\hline \multirow[t]{2}{*}{$\begin{array}{l}\mathrm{Sl} . \\
\mathrm{No}\end{array}$} & \multirow[t]{2}{*}{$\begin{array}{l}\text { Performance } \\
\text { aspects }\end{array}$} & \multicolumn{5}{|c|}{$\begin{array}{l}\text { Status Before QMS } \\
\text { Implementation }\end{array}$} & \multicolumn{5}{|c|}{$\begin{array}{c}\text { Status: Within } 3 \text { years } \\
\text { After QMS } \\
\text { implementation }\end{array}$} \\
\hline & & $\mathrm{E}$ & G & M & LM & $\mathrm{P}$ & $\mathrm{E}$ & $\mathrm{G}$ & M & LM & $\mathrm{P}$ \\
\hline & ximum Rating (B) & 100 & 80 & 60 & 20 & 0 & 100 & 80 & 60 & 20 & 0 \\
\hline 1. & $\begin{array}{l}\text { Total Items } A \\
\text { ( } 50 \text { questions in the } \\
\text { questionnaire) }\end{array}$ & 0 & 0 & 3 & 21 & 26 & 5 & 23 & 19 & 2 & 1 \\
\hline 2. & Maximum score (C) & \multicolumn{5}{|c|}{$100 \times 50=5000$} & \multicolumn{5}{|c|}{$100 \times 50=5000$} \\
\hline 3. & $\begin{array}{l}\text { Obtained score } \\
D=\Sigma(A X B)\end{array}$ & \multicolumn{5}{|c|}{600} & \multicolumn{5}{|c|}{3520} \\
\hline 4. & $\begin{array}{l}\text { \% Obtained score } \\
\text { (DX100)/C }\end{array}$ & \multicolumn{5}{|c|}{$12 \%$} & \multicolumn{5}{|c|}{$70.40 \%$} \\
\hline
\end{tabular}

Findings / Inferences: QMS Vs Organizational overall performance

Percentage Analysis shows, 58.4\% improvement in the overall organizational performance is found after QMS implementation. Chi-square test shows $P$ value $=0.000$, which is a significant change. Hence, it is interpreted that, the role of QMS has significance in improving the organizational overall performance of the organization.

\section{Summary of Findings/Inferences}

\section{Findings/Inferences: QMS Vs Financial Performance}

Percentage Analysis shows, 40\% improvement in the financial performance is found after QMS implementation. However chisquare test shows $P$ value $=0.129$ which is not a significant change. Hence, it is interpreted that, the role of QMS has no significance in improving the financial performance of the organization.

\section{Findings/Inferences: QMS Vs Productivity}

Percentage Analysis shows, 66.7\% improvement in the productivity is found after QMS implementation. Chi-square test shows $P$ value $=0.007$, which is a significant change. Hence, it is interpreted that, 
the role of QMS has significance in improving the productivity of the organization.

\section{Findings/Inferences: QMS Vs Quality Improvement}

Percentage Analysis shows, $61.3 \%$ improvement in the quality is found after QMS implementation. Chi square test shows $\mathrm{P}$ value $=0.000$, which is a significant change. Hence, it is interpreted that, the role of QMS has significance in quality improvement of the organization.

\section{Findings/Inferences: QMS Vs Employee Performance}

Percentage Analysis shows, 50\% improvement in the employee performance is found after QMS implementation. Chi-square test shows $P$ value $=0.05$, which is a significant change. Hence, it is interpreted that, the role of QMS has significance in improving the employee performance of the organization.

\section{Findings/Inferences: QMS Vs Customer Relation Management}

Percentage Analysis shows, $73.3 \%$ improvement in the customer relation management is found after QMS implementation. Chisquare test shows $\mathrm{P}$ value $=0.05$, which is a significant change. Hence, it is interpreted that, the role of QMS has significance in improving the customer relation management of the organization.

\section{Findings/Inferences: QMS Vs Organizational Cultural changes}

Percentage Analysis shows, 40\% improvement in the organizational cultural changes is found after QMS implementation. Chi-square test shows $P$ value $=0.046$, which is a significant change. Hence, it is interpreted that, the role of QMS has significance in improving the organizational cultural changes.

\section{Findings/Inferences: QMS Vs Document/Data/Record controls}

Percentage Analysis shows, 72\% improvement in the document/data/record controls is found after QMS implementation. Chi-square test shows $\mathrm{P}$ value $=0.019$, which is a high significant change. Hence, it is interpreted that, the role of QMS has significance in improving the performance related to document, data and record controls of the organization. 
Sivaramakrishnan and S Giridhara Role of Quality Management System

\section{Findings/Inferences: QMS Vs Top Management Controls}

Percentage Analysis shows, 53.3\% improvement in the top management controls is found after QMS implementation. Chisquare test shows $\mathrm{P}$ value $=0.007$, which is a significant change. Hence, it is interpreted that, the role of QMS has significance in improving the top management controls of the organization.

\section{Findings/Inferences: QMS Vs Organizational overall performance}

Percentage Analysis shows, 58.4\% improvement in the overall organizational performance is found after QMS implementation. Chi-square test shows $P$ value $=0.000$, which is a significant change. Hence, it is interpreted that, the role of QMS has significance in improving the organizational overall performance of the organization.

\section{Findings/Inferences}

Eight out of nine performance factors studied shows significant improvement. Hence the finding of the study is "The Role of Quality Management System has enhanced the performance of a Manufacturing Industry significantly".

Table 10: Summary of findings/Inferences

The interpretation of results of the analysis of the study is given in the following table

\begin{tabular}{|c|c|c|c|c|c|c|}
\hline $\begin{array}{l}\mathrm{S} \\
\mathrm{L}\end{array}$ & $\begin{array}{c}\text { Performance } \\
\text { aspect }\end{array}$ & $\begin{array}{l}\text { Befor } \\
\text { e } \\
\text { QMS } \\
\text { (A) }\end{array}$ & $\begin{array}{c}\text { After } \\
\text { QMS } \\
\text { (B) }\end{array}$ & $\begin{array}{c}\text { Improve- } \\
\text { ment \% } \\
(\mathrm{B}-\mathrm{A})\end{array}$ & $\begin{array}{c}\text { P value in } \\
\text { the } \\
\text { Chi } \\
\text { Square } \\
\text { test \# }\end{array}$ & $\begin{array}{c}\text { Interpretati } \\
\text { on and } \\
\text { Inferences } \\
\text { (To decide } \\
\text { it as } \\
\text { significant } \\
\text { P value } \\
\text { should be } \\
\text { less than } \\
\text { or equal to } \\
0.05 \text { ) }\end{array}$ \\
\hline 1. & $\begin{array}{l}\text { Financial } \\
\text { performance }\end{array}$ & 11.4 & 51.4 & 40 & $P=0.129$ & $\begin{array}{c}\text { No } \\
\text { significant } \\
\text { inference by } \\
\text { the QMS. }\end{array}$ \\
\hline 2. & Productivity & 20 & 86.7 & 66.7 & $\mathrm{P}=0.007$ & Significant \\
\hline 3. & $\begin{array}{l}\text { Quality } \\
\text { Improvement }\end{array}$ & 8 & 69.3 & 61.1 & 0.000 & Significant \\
\hline
\end{tabular}




\begin{tabular}{|c|c|c|c|c|c|c|}
\hline 4. & $\begin{array}{l}\text { Employee } \\
\text { Performance }\end{array}$ & 13.3 & 63.3 & 50 & 0.05 & Significant \\
\hline 5. & $\begin{array}{l}\text { Customer relation } \\
\text { management }\end{array}$ & 6.7 & 80 & 73.3 & 0.05 & Significant \\
\hline 6. & Cultural changes & 20 & 60 & 40 & 0.046 & Significant \\
\hline 7. & $\begin{array}{l}\text { Document/data } \\
\text { control }\end{array}$ & 12 & 84 & 72 & 0.019 & Significant \\
\hline 8. & $\begin{array}{l}\text { Top Management } \\
\text { aspects }\end{array}$ & 10 & 63.3 & 53.3 & 0.007 & Significant \\
\hline 9. & $\begin{array}{l}\text { Overall } \\
\text { performance of } \\
\text { the organization }\end{array}$ & 12 & 70.4 & 58.4 & 0.000 & Significant \\
\hline
\end{tabular}

\section{Discussions, Conclusion and Recommendations}

\section{Conclusions}

Eight out of nine performance factors studied shows significant improvement result. Hence, the finding of the study is "The Role of Quality Management System has enhanced the performance of a Manufacturing Industry significantly".

As already discussed in the literature review-1, research study by Esin Sadikoglu and Hilal, reveals that financial performance also has improved after QMS implementation. Whereas, the current study results show, that, there is no significant improvement realized by the organization in the financial performance from QMS implementation.

\section{Managerial Implications}

Based on the discussions had with the management team, the following causes are enlisted for non- achievement of financial performance...

a) There were no extra order generations due to QMS implementation alone. So, no increase is found in turnover and profits.

b) The organization was supplying all products to only one principal corporate brand customer in India. So, no increase is found in turnover and profits.

c) Only domestic market was focused. No exports were done in the past 3 years. 
d) No measurable data had been taken by the organization with respect to in the return of investment (investment made for QMS implementation) in the overall profit.

e) (e) QMS Standard is silent in the finance and account aspects and its controls. In manufacturing sector, finance/accounts processes are not covered during QMS implementation and certification audits.

\section{Discussions and Recommendations}

Employee performance is still not fully focused by the standard. ISO 9001 requirements are limited to competency and skill development part of employee but concepts like employee safety, employee motivation, employee involvement, maintaining cordial relations with employees, fulfilling employee welfare needs etc., are not covered in the standard. No requirements in the standard with regard to change management. Hence no initiatives from the organization towards change management.

Role of QMS has enhanced overall performance of an Indian Manufacturing Industry. No significant impact by QMS was found on the financial performance.

Following aspects can be covered in the ISO 9001 standard itself and these aspects are to be enforced by the consultants, organizations and certification body auditors to realize more benefits by the organizations .

1. At present, general recognition/value for ISO 9001 certificate in the market is going down, because all firms either highlyperforming-firm or non-performing-firm is getting this certificate easily. There are no stringent requirements in the standard to ensure the performance. All certified companies are treated with equality. No grading/score system between QMS of two certified organizations. For instance, in Bureau of Energy Efficiency Model of Government of India, for electrical products, Star Rating is given, based on the performance of the product. Due to these reasons, there is no pressure/vision to the organizations to perform towards achieving higher standards/goals during QMS implementation and certification process. 
2. The standard may be refined to include key financial controls and related practices. The standard may be refined to include key employee performance improvement practices. The standard may be refined to include mechanisms and best models for customer enquiry and order generation processes. Standard may be refined to include all business management related processes so that overall performance of the organization is initiated through a systematic way.

3. To improve further performance, it is recommended to finetune the standard requirements with regard to organizational improved performance. Refinements of ISO 9001 standard towards organizational performance improvement may be recommended by the organization to ISO through Bureau of Indian Standards-BIS (www.bis.org.in).

ISO 9001:2015 standard which is going to be released at the end of 2015 is expected to fulfil these needs.

\section{Scope for Future Research}

Similar to this study, the following research work may be executed.

- Effective ways to getting financial benefits from ISO 9001 (Organizations to implement additional standard already published : ISO 10014:2006)

- Role of QMS in achieving business goals

- Role of QMS in improving performance by Government organizations /Hospital/Education/Finance sector/Any other sectors

- Role of other similar standards and its performance in meeting the market requirements

- Study on number of certification obtained and withdrawals in various parts of India and world wide 
Sivaramakrishnan and S Giridhara Role of Quality Management System

\section{References}

Dale H. Besterfield, C. B. (2005). Total Quality Management. Pearson Education.

Sadikoglu, E. and Olcay, H. (2014). The Effects of Total Quality Management Practices on Performance and the Reasons of and the Barriers to TQM Practices in Turkey. Advances in Decision Sciences, 911.

Garcia, S. E. (1999). Organizational Performance and Quality Management: An Analysis of Workforce Development Organizations in Texas. The Department of Political Science, Southwest Texas State University.

Neil, J. P. (1998). Using 9000 to Go Beyond Industry Norms. Quality Progress Journal, 43-44.

Norman, N. (1999). ISO 9000, No longer a stranger to service. Quality Digest, 33-96.

Ramasamy, S. (2005). Total Quality Management. New Delhi: Tata McGrawHill.

Roman, P. (2000). ISO 9000: What is impact on Performance?. Quality Management Journal, 38-55. 